\title{
MHD simulations of accretion onto magnetized neutron star in the "propeller" regime
}

\section{Olga Toropina}

Space Research Institute (IKI)

E-mail: toropinaliki.rssi.ru

\section{Romanova}

Cornell University

E-mail: romanovalastro.cornell.edu

\section{R.V.E. Lovelace}

Cornell University

E-mail: rvllecornell.edu

We use axisymmetric magnetohydrodynamic simulations to investigate the spinning-down of magnetars rotating in the propeller regime and moving supersonically through the interstellar medium. The simulations indicate that magnetars spin-down rapidly due to this interaction, faster than for the case of a non-moving star. We discuss this model in respect of Soft Gamma Repeaters (SGRs) and the isolated neutron star candidates

25th Texas Symposium on Relativistic Astrophysics - TEXAS 2010

December 06-10, 2010

Heidelberg, Germany 


\section{Introduction}

Some neutron stars referred to as "magnetars" have unusually large magnetic fields, $B \sim$ $10^{13}-10^{15} \mathrm{G}$ [2]. Possible candidates for magnetars include anomalous X-ray pulsars and soft gamma-ray repeaters (SGRs). These objects are associated with supernovae remnants and hence are relatively young. Only a few candidates for magnetars have been found so far. The estimated birthrate of magnetars is $\sim 10 \%$ of ordinary pulsars [4] so that there might be many more magnetars which are presently invisible. Their "visibility" depends on a number of factors. One important factor is the rate of the star's spin-down. If magnetars spin-down rapidly to very long periods, then one will not detect spin modulated variability during flares.

During the pulsar stage of evolution, magnetars spin down much more rapidly than ordinary pulsars. They pass through their pulsar stage much faster, in $\sim 10^{4}$ years [9]. When the light cylinder radius becomes larger than magnetospheric radius $r_{m}$, the relativistic wind is suppressed by the inflowing matter $[8]$ and the star enters the propeller regime where the spin-down is due to the interaction of the star's rotating field with the interstellar medium (ISM) [1], [3]. Magnetars with velocities $v>100-200 \mathrm{~km} / \mathrm{s}$ interact directly with the ISM. That is, the magnetospheric radius is larger than gravitational capture radius and the rapidly rotating magnetosphere interacts strongly with the supersonically inflowing ISM.

The spin-down rate of supersonically moving magnetars in the propeller regime has been estimated earlier, but different authors have obtained rather different results. For example, Rutledge in [7] estimates a spin-down time of $\sim 4 \times 10^{9} \mathrm{yr}$ for a neutron star with a surface magnetic field $B=10^{15} \mathrm{G}$ and velocity $v=300 \mathrm{~km} / \mathrm{s}$. On the other hand, Mori and Ruderman in [5] estimate that a magnetar spins-down to periods greater than $10^{4}$ s within $\sim 5 \times 10^{5}$ years. Mori and Ruderman put forward this model as an explanation of the isolated neutron star (INS) candidate RX J1856.5-3754.

\section{Model}

We investigate the interaction of fast moving rotating magnetized star with the ISM using an axisymmetric, resistive MHD code. The code incorporates the methods of local iterations and flux-corrected-transport [13]. The flow is described by the resistive MHD equations:

$$
\begin{gathered}
\frac{\partial \rho}{\partial t}+\nabla \cdot(\rho \mathbf{v})=0 \\
\rho \frac{\partial \mathbf{v}}{\partial t}+\rho(\mathbf{v} \cdot \nabla) \mathbf{v}=-\nabla p+\frac{1}{c} \mathbf{J} \times \mathbf{B}+\mathbf{F}^{g}, \\
\frac{\partial \mathbf{B}}{\partial t}=\nabla \times(\mathbf{v} \times \mathbf{B})+\frac{c^{2}}{4 \pi \sigma} \nabla^{2} \mathbf{B} \\
\frac{\partial(\rho \varepsilon)}{\partial t}+\nabla \cdot(\rho \varepsilon \mathbf{v})=-p \nabla \cdot \mathbf{v}+\frac{\mathbf{J}^{2}}{\sigma}
\end{gathered}
$$

We assume axisymmetry $(\partial / \partial \phi=0)$, but calculate all three components of velocity and magnetic field $\mathbf{v}$ and $\mathbf{B}$. The equation of state is taken to be that for an ideal gas, $p=(\gamma-1) \rho \varepsilon$, with specific heat ratio $\gamma=5 / 3$. The equations incorporate Ohm's law $\mathbf{J}=\sigma(\mathbf{E}+\mathbf{v} \times \mathbf{B} / c)$, where $\sigma$ is the 
electrical conductivity. The associated magnetic diffusivity, $\eta_{m} \equiv c^{2} /(4 \pi \sigma)$, is considered to be a constant within the computational region. The gravitational force, $\mathbf{F}^{g}=-G M \rho \mathbf{R} / R^{3}$, is due to the central star.

We use a cylindrical, inertial coordinate system $(r, \phi, z)$ with the $z$ - axis parallel to the star's dipole moment $\mu$ and rotation axis $\Omega$. The vector potential $\mathbf{A}$ is calculated so that $\nabla \cdot \mathbf{B}=0$ at all times. The star rotates with angular velocity $\Omega_{*}=\Omega_{*} \hat{\mathbf{z}}$. The intrinsic magnetic field of the star is taken to be an aligned dipole, with vector potential $\mathbf{A}=\mu \times \mathbf{R} / R^{3}$.

More detailed description of numerical model you can see in [12].

\section{Results}

We investigated numerically interaction of the rotating magnetar with the ISM and the rate of spinning-down. Simulations were done at a variety of parameters: magnetic moments of the star $\mu$, angular velocities $\Omega_{*}$, Mach numbers $\mathscr{M}$ and diffusivities $\tilde{\eta}$. In most cases we varied one parameter in a time, and kept other parameters fixed and corresponding to the "main" case. In the main case a star rotates with an angular velocity $\omega_{*}=\Omega_{*} / \Omega_{K *}=0.7$, where $\Omega_{K *}=\sqrt{G M / R_{*}^{3}}$ is Keplerian angular velocity at the surface of the numerical star. We suggest that numerical star (inner boundary) is much larger than the true radius of the neutron star. Mach number is $\mathscr{M}=3$. We take dimensionless magnetic moment $\mu=10^{-7.5}$, and take magnetic diffusivity $\tilde{\eta}_{m}=10^{-5}$.

\subsection{Dependence of Torque on Mach Number}

For parameters corresponding to our "main case," we did simulation runs for the Mach numbers $\mathscr{M}=1,3,5,6$, and 10, with the ambient sound speed fixed. From these runs we find that the torque decreases with the Mach number approximately as $\dot{L} \propto-\mathscr{M}^{-0.4}$.

Figure 1a shows that for a Mach number $\mathscr{M}=1$ the flow is similar to that observed in case of a non-moving propeller [6]. Rapidly rotating magnetosphere pushes matter and magnetic flux outward in the equatorial plane forming the low-density, rotating torus. The gravitational radius is several times larger than magnetospheric radius so that a significant part of the inflowing matter is gravitationally trapped and accumulates around the star. This is similar to the case of spherical Bondi accretion to a star in the propeller stage. For $\mathscr{M}=1$, there is an axial flow of matter downstream from the shock wave. However, the energy-density of the inflowing matter $\rho \mathbf{v}^{2} / 2$ is smaller than the energy-density of the equatorial propeller outflow $\rho \mathbf{v}^{2} / 2+\mathbf{B}^{2} / 8 \pi$ and this is why the equatorial structure forms.

Figure $1 \mathrm{~b}$ shows the flow at a larger Mach number $\mathscr{M}=3$. In this case the energy-density of the ISM matter is larger than the energy-density of the equatorial propeller-generated wind, so that the disk structure is bent and pushed to the direction of the tail. This interaction is similar to that observed in the simulations of magnetized supersonic stars in the non-rotating case [10].

The magnetosphere of the star acts as an obstacle for the ISM matter so that a bow shock stands in front of the star and a conical shock wave forms behind it. The stand-off distance and the cross-section of the interaction is larger in the propeller case than in the non-rotating case. This is because the rotating equatorial disk of matter and magnetic field generated by the fast rotating magnetosphere. For Mach numbers $\mathscr{M}>2-3$, this disk structure is pushed by the inflowing matter into a magnetotail behind the star. 

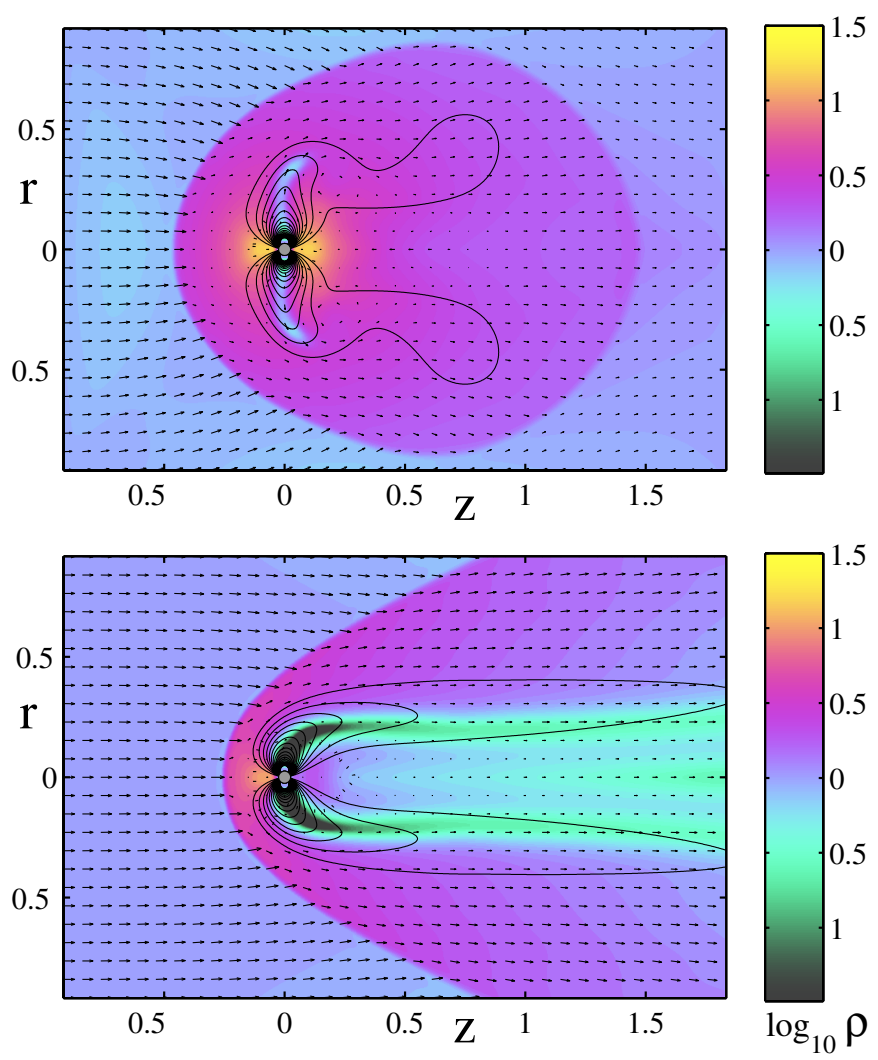

Figure 1: Matter flow around a strongly magnetized star rotating in the propeller regime and propagating through the interstellar medium with Mach numbers $\mathscr{M}=1$ (top panel) and $\mathscr{M}=3$ (bottom panel).

For even larger Mach numbers, $\mathscr{M}=6-10$, the flow is similar to that observed for $\mathscr{M}=3$. However, the stand-off distance is even smaller, because the larger portion of the rotating magnetosphere is pushed into the magnetotail behind the star.

The rotating magnetosphere interacts with the non-rotating matter of the ISM and this leads to the spinning-down of the star. Angular momentum lost by the star flows out from the star. Thus, we calculate the torque on the star by integrating the angular momentum flux density over a surface surrounding the star,

$$
\dot{L}=-\int d \mathbf{S} \cdot\left(\rho \mathbf{v}_{p} r v_{\phi}-\frac{\mathbf{B}_{p} r B_{\phi}}{4 \pi}\right)
$$

Here, $d \mathbf{S}$ is the outward pointing surface area element and the $p$-subscript indicates the poloidal component. We use a cylindrical surface around the star approximately at the Alfvén surface to evaluate this integral.

Figure 2 shows temporal variation of this flux. After few initial rotations of the star, $\dot{L}$ becomes approximately constant. For comparison, we also calculated angular momentum flux through the magnetotail at the distance $z=0.6$ from the star. We obtained similar value of the angular momentum flux (dashed line) because the angular momentum lost by the star flows into the magnetotail.

Figure 3 shows distribution of angular momentum flux densities carried by the magnetic field $-\mathbf{B}_{p} r B_{\phi} / 4 \pi$ (top panel) and that carried by the matter $\rho \mathbf{v}_{p} r v_{\phi}$ (bottom panel). One can see that in- 


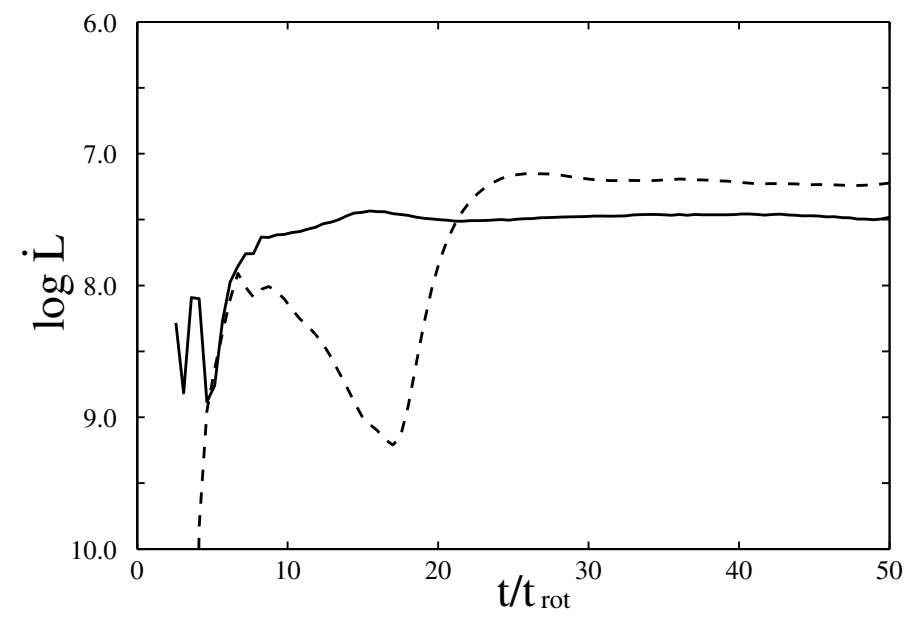

Figure 2: Variation of the total angular momentum flux from the star over a surface surrounding the star (solid line) and the flux through a surface across the magnetotail at $z=0.6$ (dotted line)

side the star's magnetosphere the magnetic field gives the main contribution to the angular momentum flux (see top panel). After passing the shock wave, magnetosphere interacts with non-rotating ISM and transports part of its angular momentum to the matter. This rotating matter propagating to the magnetotail gives the main contribution to the angular momentum flux in the tail (see bottom panel). The rate of angular momentum loss from the star depends on efficiency of mixing of the ISM matter with the magnetic field of the magnetosphere.

\subsection{Dependence of the Torque on Other Parameters}

We performed a number of simulations with different angular velocities of the star $\Omega_{*}$ and different magnetic moments $\mu$. We calculated the total angular momentum loss rate from the star and investigated its dependence on the $\mathscr{M}, \Omega_{*}, \mu$ and $\tilde{\eta}_{m}$. In addition we calculated the dependence of the torque on the density of the ISM medium. We obtain the summary of scaling laws:

$$
\frac{d L}{d t} \propto-\eta^{0.3} \mu^{0.6} \rho^{0.8} \mathscr{M}^{-0.4} \Omega_{*}^{1.5}
$$

\section{Spinning Down of Magnetars}

We can estimate spinning down of magnetars in real, dimensional units. We take as a base our main dimensionless parameters: angular velocity $\Omega / \Omega_{K^{*}}=0.7$, Mach number $\mathscr{M}=3$, gravimagnetic parameter $\beta=10^{-6}$ and magnetic diffusivity $\tilde{\eta}_{m}=10^{-5}$.

We consider a neutron star with mass $M=1.4 M_{\odot}=2.8 \times 10^{33} \mathrm{~g}$ and radius $R_{N S}=10^{6} \mathrm{~cm}$. The density of the ambient interstellar matter is taken to be $\rho=1.7 \times 10^{-24} \mathrm{~g} / \mathrm{cm}^{3}\left(n=1 / \mathrm{cm}^{3}\right)$. The sound speed in the ISM, $c_{s}=30 \mathrm{~km} / \mathrm{s}$ and a star moves in the interstellar medium with velocity $v=3 c_{s}=90 \mathrm{~km} / \mathrm{s}$.

Using those values we can obtain:

$$
\dot{L} \approx 3 \times 10^{29} n_{1} c_{30}^{-4} B_{15}^{0.6} P_{5}^{-1.5} \mathscr{M}_{3}^{-0.4} \mathrm{~g}(\mathrm{~cm} / \mathrm{s})^{2} .
$$



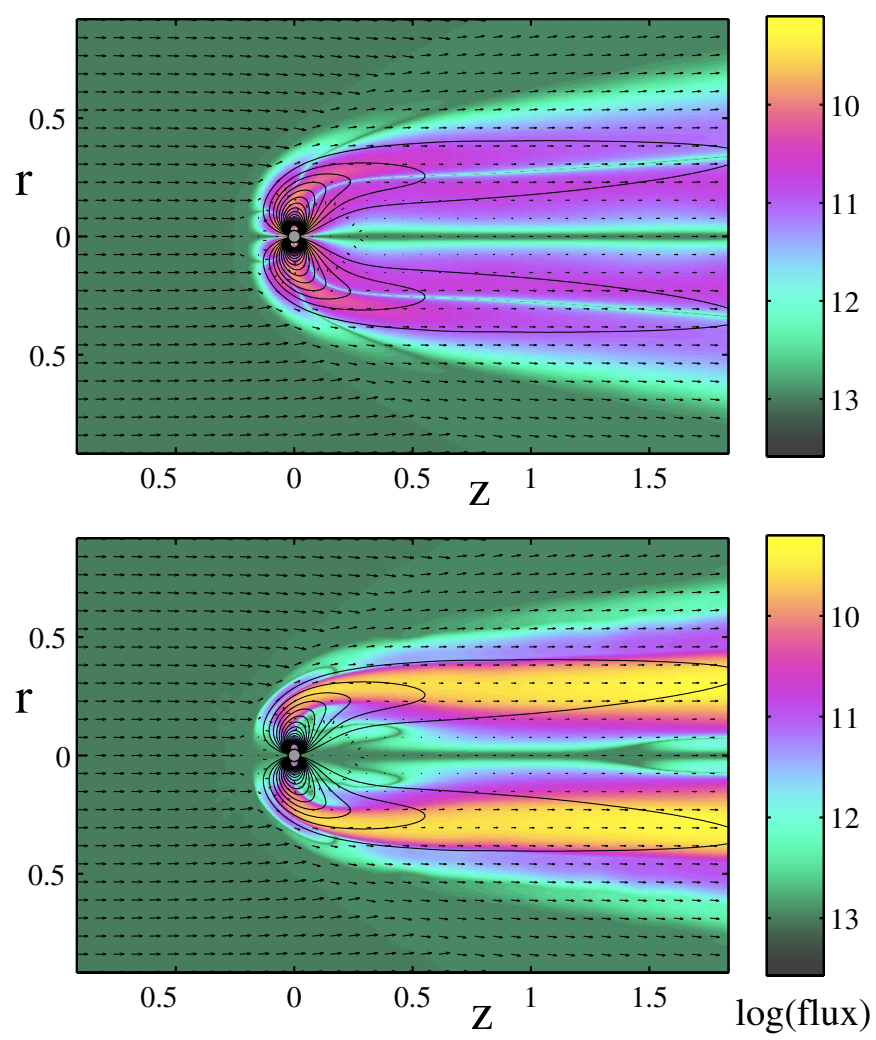

Figure 3: Distribution of the angular momentum fluxes in the magnetotail.

Thus the characteristic spin-down time is

$$
T=L_{*} /|\dot{L}| \approx 10^{4} n_{1}^{-1} c_{30}^{4} B_{15}^{-0.6} P_{5}^{0.5} \mathscr{M}_{3}^{0.4} \mathrm{yr} .
$$

Next we can estimate the time-scale of evolution at the propeller stage. For periods $P_{*}=10^{3} \mathrm{~s}$, which correspond to beginning of the propeller stage, the evolution scale will be $\Delta T=10^{3}$ years, while at period $P_{*}=10^{6} \mathrm{~s}$ corresponding to the end of propeller stage $T=3 \times 10^{4}$ years. Thus we see that magnetars are expected to spin down very fast at the propeller stage. This time-scale however may be much larger if diffusivity is very small.

\section{Conclusions}

Using axisymmetric MHD simulations we have studied the supersonic propagation through the ISM of magnetars in the propeller stage. We have done many simulation runs for the purpose of determining the angular momentum loss rate of the star due to the interaction of its magnetosphere with the shocked ISM. We conclude, that the interaction may be highly effective in spinningdown magnetars. A star with magnetic field $B \sim 10^{13}-10^{15} \mathrm{G}$ is expected to spin-down in $\Delta T \sim$ $10^{4}-10^{5}$ years. This time may be longer if the ISM material does not efficiently interact with the external regions of the magnetar's magnetosphere. Therefore, after relatively short stages of pulsar and propeller activity, a magnetar becomes a very slowly rotating object, with a period 
$P>10^{5}-10^{6} s$, which is much longer than the periods expected for ordinary pulsars. This may be a reason why the number of soft gamma repeaters, which are candidate magnetars, is so small. We should note however, that the rate of spinning-down depends on the magnetic diffusivity which is not known. At lower diffusivity the rate of spinning-down will be lower. The INS candidate RX J1856.5-3754 may be an example of a slowly rotating magnetar. However, this model does not explain the $H_{\alpha}$ nebulae. An ordinary misaligned pulsar explains the different features more easily, excluding the fact that no periodic fluctuations were observed from this object.

\section{References}

[1] K.Davidson \& J.P. Ostriker, 1973, ApJ, 179, 585

[2] R.C. Duncan \& C. Thompson, 1992, 392, L9

[3] A.F. Illarionov \& R.A. Sunyaev, 1975. A\&A, 39, 185

[4] S.R. Kulkarni \& D.A. Frail, 1993, Nature, 365, 33

[5] K. Mori, M.A. Ruderman, 2003, ApJ, 592, L75

[6] M.M. Romanova, O.D. Toropina, Yu.M. Toropin \& R.V.E. Lovelace, 2003, ApJ, 588, 400

[7] R.E. Rutledge, 2001, ApJ, 553, 796

[8] V.F. Shvartsman 1970, Radiofizika, 13, 1852

[9] C. Thompson \& R.C. Duncan, 1995, MNRAS, 275, 255

[10] O.D. Toropina, M.M. Romanova, Yu.M. Toropin \& R.V.E. Lovelace, 2001, ApJ, 561, 964

[11] O.D. Toropina, M.M. Romanova, Yu.M. Toropin \& R.V.E. Lovelace, 2003, ApJ, 593, 472

[12] O.D. Toropina, M.M. Romanova \& R.V.E. Lovelace, 2006, MNRAS, Volume 371, 569

[13] V.T. Zhukov, A.V. Zabrodin \& O.B. Feodoritova, 1993, Comp. Maths. Math. Phys., 33, No. 8, 1099 